\title{
ON THE CONTINUITY OF IMAGES BY TRANSMISSION IMAGING*
}

\author{
CHUNLIN WU $\mathrm{WU}^{\dagger}$
}

\begin{abstract}
Transmission imaging is an important imaging technique which is widely used in astronomy, medical diagnosis, and biology science, whose imaging principle is quite different from that of reflection imaging used in our everyday life. Images by reflection imaging are usually modeled as discontinuous functions and even piecewise constant functions in most cases. This discontinuity property is the basis for many successful image processing techniques such as the popular total variation (TV) regularization. In this paper we prove that almost all images by transmission imaging are continuous functions. For the convenience of description, we will consider transmission imaging with parallel line geometry of wave beam, which is a fundamental geometry in transmission imaging and has been extensively applied in microscopes. In this kind of imaging, people take images of the physical scene from many different projection directions. We will prove that for almost every projection direction, the generated image is a continuous function, even if the density function of the physical scene is discontinuous. If the density functions of the objects to be imaged are radial regardless of some coordinate shifts, then all the projection directions generate continuous images. This continuity property has not been published yet in the literature. As a straightforward application, we finally present a simple yet effective improvement of TV regularization for Poisson noise (which is the most significant noise in transmission imaging) removal. Numerical examples and comparisons verify our analysis and demonstrate the effectiveness of the improved model.
\end{abstract}

Key words. Transmission imaging, reflection imaging, Radon transform, parallel line geometry, continuity, measure zero, radial function, Poisson noise, variational method.

AMS subject classifications. 92C55, 90C90, 68U10.

\section{Introduction}

Imaging is an important technique which translates a physical scene to lower dimensional (typically 2D) data for convenient observation and record. It has been applied to many fields, including our everyday life, medical diagnosis, exploring the universe, and biological structure analysis. Many imaging systems and instruments, such as various digital cameras, X-ray CT, telescopes, and microscopes, have been developed. Different imaging systems are based on different physical principles. Digital cameras used in our everyday life record the reflection part of the white light from objects [22] except when capturing the sky, whereas transmission electron microscopes generate images by counting the electrons having transmitted the scene $[18,11,23]$. We refer to these two kinds of imaging techniques by reflection imaging and transmission imaging in this paper for clarity.

As is well known, images by reflection imaging are usually modeled as discontinuous functions and even piecewise constant functions in most cases. Consequently most of them have sparse gradients. Due to this property, many image processing techniques, such as the popular total variation (TV) regularization [29], have achieved great successes.

Although the central topic in transmission imaging is the reconstruction of the 3D objects from their 2D projection data (which are our so-called images) $[4,11,16,24$, 34], restoration of these data (before $3 \mathrm{D}$ reconstruction) is sometimes also important due to the involvement of noise and other degradations during the imaging procedure $[37,25,28,38]$. A typical problem is how to remove the Poisson noise (which is the

\footnotetext{
${ }^{*}$ Received: June 28, 2011; accepted (in revised form): July 23, 2012. Communicated by Luminita Vese.

${ }^{\dagger}$ Department of Mathematics, National University of Singapore, Block S17, 10, Lower Kent Ridge
} Road, Singapore 119076. 
dominant noise) in images by transmission imaging. Very recently, many scholars studied the application of TV regularization to this ill-posed problem [10, 20, 12, 32, $40,42]$ and piecewise constant images were widely used in their numerical tests.

In this paper we show that almost all images by transmission imaging are actually continuous and consequently TV regularization for this kind of images can be greatly improved. For convenience of description and consistency of notation, we consider transmission imaging with parallel line geometry of wave beam, which is a fundamental beam geometry $[18,23]$ and has been extensively used in microscopes [11]. In transmission imaging (with parallel line geometry), people take images (also called projections in the literature) from many different projection directions in order to reconstruct the density functions of the imaged objects. Each projection direction corresponds to one image. We will prove that for almost every projection direction, the generated image is a continuous function, even if the density functions of the imaged objects are discontinuous (discontinuous density functions are very common). The set of projection directions generating discontinuous images has measure zero. If the density functions of the objects to be imaged are radial regardless of some coordinate shifts, then all the projection directions will generate continuous images. As is well known, the Radon transform [27] is the essential mathematical tool to describe the imaging procedure. As far as we know, theoretical results on the Radon transform in the literature focus on the analysis of the imaging procedure as a mapping operator $[15,18,23]$, e.g., the invertability of the operator. In addition, most analyses assume that the density function of the object to be imaged is a continuous or even Schwartz function all over the Euclidean space [15, 23, 27]. So far our analysis has not appeared in the literature, although discontinuous density functions of objects are very common and thus important in applications. As a consequence of our results, most current digital image processing techniques, which are based on the discontinuities (edges) of the image, may not be best for images generated via transmission imaging. The popular TV regularization for Poisson noise removal $[4,10,20,12,24,32,40,42]$ can be greatly improved. We will finally present a simple variational approach for Poisson noise removal by appropriately combining some existing regularization and fidelity terms, which improves the TV regularization based methods $[4,10,20,12,24,32,40,42]$ dramatically. The regularization term to be used is a high order generalization of TV. Recently many high order generalizations of TV have been proposed in the context of reducing staircase effect or image decomposition $[7,21,30,35,41,17,26,6,8,31,33,3,19]$. Please see Section 4 for a review and classification of them.

The paper is organized as follows. To better understand the background of our analysis, we will present in Section 2 the principles of reflection imaging and transmission imaging and brief comparisons between the features of images generated by them. In Section 3, we will focus on the continuity problem of images taken via transmission imaging. A simple yet effective improvement of the most popular TV regularization for Poisson noise removal will be presented in Section 4, verifying our analysis and showing a straightforward application of our theoretical results. The paper is concluded in Section 5.

\section{Principles of reflection imaging and transmission imaging}

As indicated by the terms, reflection imaging relies on the reflection of the light wave on the surface of the objects [22], whereas transmission imaging is based on some radial wave (such as electron wave or X-ray) arriving at the image plane by transmitting the physical scene $[18,11,23]$; see figure 2.1. For simplicity, we here focus on the 
essentially different parts of these two imaging procedures and omit other common parts (such as focus and magnification subsystems). In the following we will explain the principles of these two imaging procedures in both physical and mathematical points of view with brief comparisons. The principle and implementation of an imag-

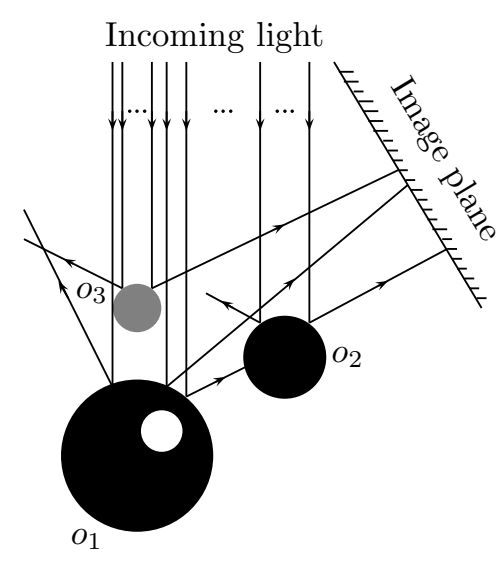

(a) reflection imaging

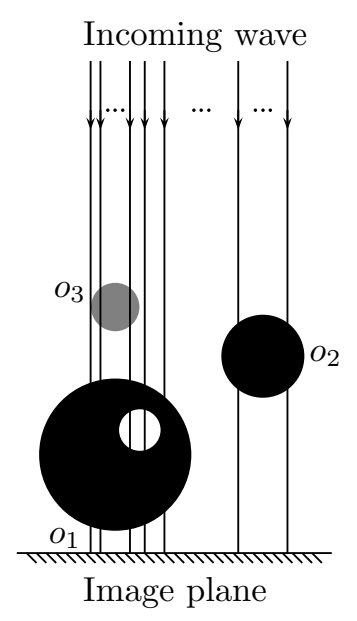

(b) transmission imaging

FIG. 2.1. A simple illustration of reflection and transmission imaging

ing system depends on the physical properties of both the objects to be imaged and the source (e.g., visible light, X-ray, and electron beam) used for imaging. Usually the source produces a composition of waves with frequencies in a certain range (including the case of just one single frequency). Waves with different frequencies have different reflection and transmission abilities. The visible light in our everyday life has lower frequencies and thus is difficult to transmit most objects. When it hits most objects, a portion of it reflects while the remainder is absorbed by the objects. Different objects can absorb waves with different frequencies. Consequently the reflected waves have different frequencies. We do recognize objects by these reflected waves, which exhibit their colors. See figure 2.1 (a), where $o_{1}, o_{2}, o_{3}$ are three objects and the image plane records the reflection light from $o_{1}, o_{2}, o_{3}$. Note that not all the reflection light of an object can be observed by the image plane, since some portions of the reflection light may be blocked by other objects.

The situation is totally different in transmission imaging case. Transmission imaging adopts the strong transmission ability of the source, e.g., $\gamma$ radial, $\mathrm{X}$-ray, and high speed electron beams, whose frequencies are much higher. These kinds of imaging techniques are mostly applied to medical diagnosis and biological structure analysis $[18,11]$. Ideally, the source produces a wave with a single frequency during one single measurement. When hitting a medical or biological scene (e.g., some biological tissue or specimen), the wave transmits the objects and then arrives at the image plane. During the transmission, the wave interacts with the objects. Usually this interaction is very complicated and very hard to precisely describe, especially in micro structure analysis at molecular or atomic level. Figure 2.1 (b) is an illustration, where $o_{1}, o_{2}, o_{3}$ are three (biological) objects and the image plane records the electrons successfully transmitting the objects. As mentioned before, here we consider transmission imaging with parallel line geometry of the wave beam $[18,11,23]$. Some other types of 
geometry such as fan beam are introduced in $[18,23]$.

In the following, we explain these two imaging procedures from the mathematical point of view. As shown in figure 2.1, we assume three objects $o_{1}, o_{2}, o_{3}$ to be imaged. We represent them via their density functions $\rho_{1}(\mathbf{r}), \rho_{2}(\mathbf{r}), \rho_{3}(\mathbf{r})\left(\mathbf{r} \in \mathbf{R}^{3}\right)$. These functions can be mass density, electron density, or others of the objects, depending on by which density the objects will interact with the source wave.

In the reflection imaging case, the light wave hits the objects and reflects. The reflection depends on the material and the position of the outer boundary of the object. (Note that we can always assume that the material of a single object is nearly homogeneous; otherwise we can further decompose it.) Each object will reflect waves with some particular small range of frequencies (even particular single frequency in many cases), which indicate the colors of the object. The direction of the reflected wave and within which domain of the image plane the wave will arrive at depend on the position of the outer boundary of the object. The inner boundary of an object (see $o_{1}$ ), and actually its whole interior body, have no effect on the reflection. Therefore, if we consider only $o_{1}$ and remove $o_{2}, o_{3}$ from the scene, an intensity function, which is nearly constant within its domain and usually discontinuous at the boundary, will be recorded on the image plane. Suppose that the intensity function generated by $o_{1}$ is $I^{r}\left(o_{1} ; r\right)$. Similarly, we have $I^{r}\left(o_{2} ; r\right)$ and $I^{r}\left(o_{3} ; r\right)$. Here the super script $r$ means "reflection imaging" and the argument $r$ is the coordinate in the image plane. Now, we consider the whole scene. If no object is warded off by others, then the total intensity in the image plane is simply a linear combination:

$$
I^{r}\left(o_{1} ; r\right)+I^{r}\left(o_{2} ; r\right)+I^{r}\left(o_{3} ; r\right) .
$$

However, in most scenes some objects are warded off by others. A portion of the reflection light of the warded object is blocked. The image plane records the sum of the blocked reflection light, which reads

$$
I^{r}\left(o_{1}+o_{2}+o_{3} ; r\right)=b_{1}(r) I^{r}\left(o_{1} ; r\right)+b_{2}(r) I^{r}\left(o_{2} ; r\right)+b_{3}(r) I^{r}\left(o_{3} ; r\right),
$$

where $b_{i}(r), i=1,2,3$ are some truncation functions indicating the block effect. $b_{i}, i=$ $1,2,3$ depend on the positions of the outer boundaries of the objects and are actually indicator functions. Therefore, we have the following conclusion:

- As an operator, reflection imaging is in general nonlinear with respect to the objects in the scene.

Since the functions $I^{r}\left(o_{i} ; r\right), \forall i$ are piecewise smooth and usually discontinuous at the boundaries of their supports, and the functions $b_{i}(r), \forall i$ are piecewise constant (thus discontinuous), $I^{r}\left(o_{1}+o_{2}+o_{3} ; r\right)$ is piecewise smooth and discontinuous at the common boundaries between objects. In most cases, the image is piecewise constant. This is exactly the classic mathematical modeling of reflection images in [22]. Nevertheless, we conclude that:

- In reflection imaging, the image is a discontinuous function, unless a single object is imaged with no background (this case seldom happens). In most cases, it is piecewise constant.

This is a very important property, based on which many image processing and segmentation techniques, models, and algorithms have been proposed in the literature.

Now let us turn to the transmission imaging; see figure 2.1 (b). In transmission imaging, the source wave transmits the scene (such as some biological specimen) and a portion of the wave arrives at the image plane. The information recorded in the 
image plane is then used to infer the structure of the scene. The interaction between the wave and the objects in the scene depends on some certain density functions of the objects. Mathematically this procedure can be modeled by the Radon transform [27]. For convenience of description, we assume the direction of the wave beam in figure $2.1(\mathrm{~b})$ is $z$. Let us first consider $o_{1}$ and remove $o_{2}, o_{3}$ from the scene. The image generated by $o_{1}$ is as follows

$$
I^{t}\left(o_{1} ; r\right)=\int \rho_{1}(\mathbf{r}) d z
$$

where we use the super script $t$ to denote "transmission imaging". (Physically speaking, the intensity sensed in the image plane is actually $C-I^{t}\left(o_{1} ; r\right)$ with a constant $C$ indicating the strength of the source wave. In mathematical modeling and analysis, there is no difference between them.) Putting $o_{1}, o_{2}, o_{3}$ all together in the scene, the total density function is $\rho_{1}(\mathbf{r})+\rho_{2}(\mathbf{r})+\rho_{3}(\mathbf{r})$, since in many cases we can omit the interaction between these objects. Therefore, the total intensity sensed in the image plane is

$$
I^{t}\left(o_{1}+o_{2}+o_{3} ; r\right)=\int \rho_{1}(\mathbf{r})+\rho_{2}(\mathbf{r})+\rho_{3}(\mathbf{r}) d z=I^{t}\left(o_{1} ; r\right)+I^{t}\left(o_{2} ; r\right)+I^{t}\left(o_{3} ; r\right) .
$$

Hence we conclude:

- As an operator, transmission imaging is linear with respect to the objects in the scene for given projection direction.

In the following section we focus on analyzing the features of $2 \mathrm{D}$ images (projections) recorded by the image plane. As mentioned previously, in transmission imaging with parallel line geometry, people take images from many projection directions. Each projection direction corresponds to an image. We will show that most images generated in transmission imaging can be modeled as continuous functions, even if the density functions of objects are discontinuous. This is totally different from reflection imaging.

\section{The continuity property of images generated by transmission imag-} ing

In this section, we study the continuity property of images taken by transmission imaging with parallel line geometry of wave beam. We will discuss the topic in two cases, which are very common in real applications. In the first case compactly supported density functions are considered. Real applications with density functions such as mass density fall into this case. We will prove that for almost every projection direction, the image taken in this direction is a continuous function. The set of discontinuous images corresponds to a set of projection directions which has measure zero. In the second case we consider radial density functions (not restricted to be compactly supported). We will show that in this case, all images from all the projection directions are continuous functions. All of these results will be presented in general Euclidean space $\mathbf{R}^{n}$ (typically $n=2,3$ ).

We first give some notation. We use $B_{\mathbf{r}, \delta}$ to denote the open ball centered at $\mathbf{r}$ with radii $\delta . S^{n-1}$ is denoted for the $n-1$ dimensional unit sphere. Its upper part is denoted by

$$
S_{+}^{n-1}=\left\{\mathbf{r}=\left(r_{1}, r_{2}, \cdots, r_{n}\right) \in \mathbf{R}^{n}:|\mathbf{r}|=1, r_{n}>0\right\} .
$$


A straight line $l \subset \mathbf{R}^{n}$ is expressed as

$$
\mathbf{r}=\mathbf{r}_{0}+\mathbf{v} t,-\infty<t<+\infty
$$

where $\mathbf{r}_{0} \in \mathbf{R}^{n}$, and $\mathbf{v} \in \mathbf{R}^{n}$ is a unit direction vector, i.e., $|\mathbf{v}|=1$. For simplicity, we denote a straight line determined by $\mathbf{r}_{0}$ and $\mathbf{v}$ as $l_{\mathbf{r}_{0}, \mathbf{v}}$ in the following.

In transmission imaging, people take images of objects from different projection directions by computing the integrals of the density of the objects along straight lines with those directions. Regardless of the symmetry of direction, we need only to consider those images generated in the projection directions, which are elements of one half of the $n-1$ dimensional sphere denoted by

$$
\begin{aligned}
S_{\frac{1}{2}}^{n-1}=\left\{\mathbf{v} \in \mathbf{R}^{n}:|\mathbf{v}|=1,\left(\theta_{1}, \theta_{2}, \cdots, \theta_{n-1}\right) \in[0, \pi]^{n-2} \times[0, \pi),\right. \\
\left.\left(\theta_{1}, \theta_{2}, \cdots, \theta_{n-1}\right) \text { are the Euler angles of } \mathbf{v}\right\}
\end{aligned}
$$

For each $\mathbf{v}$ in $S_{\frac{1}{2}}^{n-1}$, people take one image from that projection direction. We denote the image generated by $\mathbf{v} \in S_{\frac{1}{2}}^{n-1}$ as $I_{\mathbf{v}}^{t}$ (we remind that the super script $t$ means "transmission imaging"). If there are no repeated images, the images are as many as the points in $S_{\frac{1}{2}}^{n-1}$.

3.1. Compactly supported density functions. Before presenting our results, we give an example in $\mathbf{R}^{2}$; see figure 3.1. Here the object to be imaged is a rectangle $o$, whose density function is supported in the rectangle and is constant $\rho(\mathbf{r}) \equiv 0.5$. In transmission imaging, the object is projected from different directions to the corresponding image planes. The projection from each direction generates one image. In $\mathbf{R}^{2}$, the projection direction is $\mathbf{v} \in S_{\frac{1}{2}}^{1}$, or, equivalently, can be described by an azimuthal $\theta \in[0, \pi)$. That is, the unit vectors indicating the projection directions are on a half circle. In figure 3.1 , we only show two projection directions and the corresponding images of the object. As one can see, the image in figure 3.1 (a) is continuous over the whole image plane ( $\mathbf{R}^{1}$ here), whereas the image in figure 3.1 (b) is discontinuous. This example shows that different projection directions may generate images with different smoothness, even for the object of constant density. In this example, there are in total 2 projection directions (parallel to the edges of the rectangle respectively) which generate discontinuous images. In the following, we will prove that the set of projection directions generating discontinuous images has measure zero within the set of all the projection directions. We will present our results in general Euclidean space $\mathbf{R}^{n}$. By the linearity of the transmission imaging operator, we only need to consider just one single object. We denote it by $o$, whose density function is $\rho(\mathbf{r})$. The support of $\rho(\mathbf{r})$ is $D$ (singly connected because of one single object) with boundary $B$. In many real applications, we can assume: 


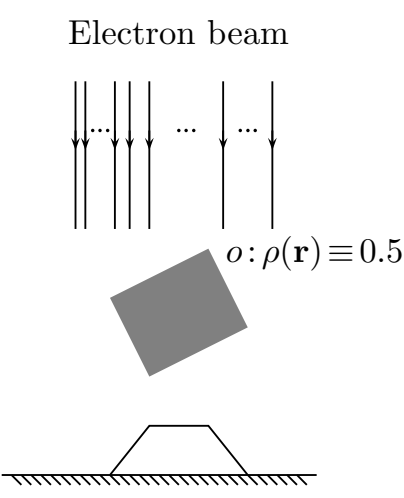

Image plane

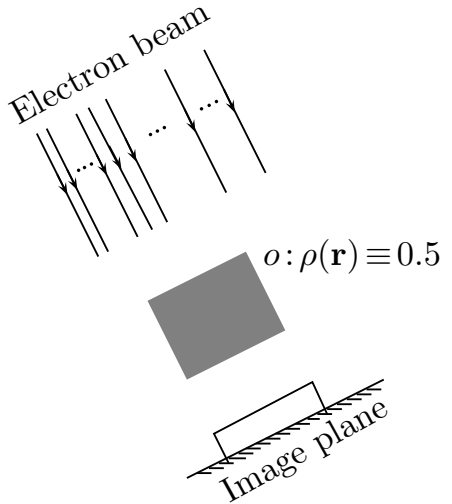

(a) a continuous projection of a rectangle

(b) a discontinuous projection of a rectangle

FIG. 3.1. An example of transmission imaging in $\mathbf{R}^{2}$.

Assumption $1 \quad \rho(\mathbf{r})$ is compactly supported, i.e., $D$ is bounded;

Assumption $2 \rho(\mathbf{r})$ is continuous over $D$;

Assumption $3 \quad B$ is a simple, continuous, and piecewise $C^{1}$ hypersurface;

Assumption $4 \quad$ For any straight line $l$ in $\mathbf{R}^{n}, l$ intersects $B$ at either empty set, or finite number of points, or finite number of line segments, or the union of finite number of points and finite number of line segments.

Assumption 5 Let $P_{n-1}$ be an $n-1$ dimensional domain of an $n-1$ dimensional hyperplane $H_{n-1}$ such that $P_{n-1}=H_{n-1} \cap B$ and $\forall p \in\left(H_{n-1} \cap B\right) \backslash P_{n-1}, P_{n-1} \cup\{p\}$ is not a domain of $H_{n-1}$. That is, $P_{n-1}$ is a locally largest $n-1$ dimensional affine patch of $B$. We denote the set of such patches by $\mathfrak{P}_{n-1}$. We similarly and recursively define sets $\mathfrak{P}_{n-2}, \mathfrak{P}_{n-3}, \cdots, \mathfrak{P}_{1}$, with the requirement that $\forall P_{d} \in \mathfrak{P}_{d}$ and $P_{d+1} \in \mathfrak{P}_{d+1}, m_{d}\left(P_{d} \cap\right.$ $\left.\operatorname{cl}\left(P_{d+1}\right)\right)=0$ where $\operatorname{cl}(\cdot)$ is the closure and $m_{d}$ is $d$ dimensional measure, $d=n-2, \cdots, 1$. As one can see, all the line segments on $B$ are on the affine patches in $\mathfrak{P}=$ $\mathfrak{P}_{n-1} \cup \mathfrak{P}_{n-2} \cup \cdots \cup \mathfrak{P}_{1}$. It is obvious that there may be parallel affine patches in $\mathfrak{P}$. For each $d=1,2, \cdots, n-1$, we further modify $\mathfrak{P}_{d}$ by keeping only one affine patch of each class of parallel affine patches. The new set is still denoted by $\mathfrak{P}_{d}, d=1,2, \cdots, n-1$, and $\mathfrak{P}_{n-1} \cup \mathfrak{P}_{n-2} \cup \cdots \cup \mathfrak{P}_{1}$ by $\mathfrak{P}$. The new sets have the same unit vectors on affine patches as before, respectively. Let $\mathfrak{E}_{d}=\left\{P_{d}: P_{d} \in\right.$ $\mathfrak{P}_{d}, P_{d} \bigcap \operatorname{cl}\left(\bigcup_{P \in \mathfrak{P} \backslash\left\{P_{d}\right\}} P\right)$ are dense on $\left.P_{d}\right\}, d=1,2, \cdots, n-$ 1. We assume that $\mathfrak{E}_{d}, d=1,2, \cdots, n-1$ are all finite sets.

We mention that the above assumptions are actually quite mild in real applications, although they look a little bit complicated. A common example is that $D$ is a polyhedron and $\rho(\mathbf{r})$ is constant over $D$. 
Lemma 3.1. Assume that $g$ is the metric tensor of the mapping $\varphi$ from the $n-1$ dimensional open ball $B_{\mathbf{0}_{n-1}, 1}\left(\mathbf{0}_{n-1}\right.$ is the origin of $\left.\mathbf{R}^{n-1}\right)$ to $S_{+}^{n-1}$ defined by

$$
\left(v_{1}, v_{2}, \cdots, v_{n-1}\right) \rightarrow\left(v_{1}, v_{2}, \cdots, v_{n-1}, v_{n}\right)=\left(v_{1}, v_{2}, \cdots, v_{n-1}, \sqrt{1-\sum_{1 \leq i \leq n-1} v_{i}^{2}}\right) .
$$

Then $\operatorname{det} g=\frac{1}{1-\sum_{1 \leq i \leq n-1} v_{i}^{2}}=\frac{1}{v_{n}^{2}}$.

Proof. By basic calculation we have

$$
g=I+V,
$$

where $I$ is the identity matrix and $V=\left(v_{i j}\right)$ with elements $v_{i j}=\frac{v_{i} v_{j}}{v_{n}^{2}}$. From linear algebra, we know

$$
\operatorname{det} g=1+\sum_{1 \leq k \leq n-1} \sum_{i_{1}<i_{2}<\cdots<i_{k}} V\left(\begin{array}{llll}
i_{1} & i_{2} & \cdots & i_{k} \\
i_{1} & i_{2} & \cdots & i_{k}
\end{array}\right)
$$

where $V\left(\begin{array}{cccc}i_{1} & i_{2} & \cdots & i_{k} \\ i_{1} & i_{2} & \cdots & i_{k}\end{array}\right)$ is a principal minor of $V$. It is easy to see that all the principal minors with orders greater than 1 have zero determinant. Therefore,

$$
\operatorname{det} g=1+\sum_{i_{1}} V\left(\begin{array}{l}
i_{1} \\
i_{1}
\end{array}\right)=\frac{1}{v_{n}^{2}},
$$

which completes the proof.

Based on Lemma 3.1, we can prove the following result.

TheOREM 3.2. Assume $Z=\left\{\mathbf{v} \in S_{\frac{1}{2}}^{n-1}: \exists \mathbf{r}_{0}\right.$, s.t. $\left.m\left(l_{\mathbf{r}_{0}, \mathbf{v}} \cap B\right)>0\right\}$ where $m\left(l_{\mathbf{r}_{0}, \mathbf{v}} \cap B\right)$ is the Lebesgue measure in $\mathbf{R}^{1}$ (along the straight line). Then $Z$ has zero spherical measure.

Proof. By restricting the direction vector on $S_{\frac{1}{2}}^{n-1}$, we know that $Z$ is nothing but the set of unit direction vectors of the line segments in the affine patches in $\mathfrak{P}$ defined in Assumption 5, i.e., $Z=\mathbf{v}(\mathfrak{P})$, by denoting all the unit direction vectors of line segments in affine patches in $\mathfrak{P}$ as $\mathbf{v}(\mathfrak{P})$.

We now construct another set $\tilde{\mathfrak{P}}$, without affecting the unit direction vectors of the line segments in the affine patches in $\mathfrak{P}$. Recall that

$$
\mathfrak{P}=\mathfrak{P}_{n-1} \cup \mathfrak{P}_{n-2} \cup \cdots \cup \mathfrak{P}_{1} .
$$

We first consider $\mathfrak{P}_{1}$. We have

$$
\mathfrak{P}_{1}=\left(\mathfrak{P}_{1} \backslash \mathfrak{E}_{1}\right) \cup \mathfrak{E}_{1} \triangleq \mathfrak{Q}_{1} \cup \mathfrak{E}_{1} .
$$

From Assumption 5, for any $P_{1} \in \mathfrak{Q}_{1}$ (actually a line segment in this case), there exists a closed 1D neighborhood $P_{1}^{\prime} \subset P_{1}$ (with positive 1D measure) such that no intersection between $P_{1}$ and the closure of the union of all the other affine patches in $\mathfrak{P}$ lies on $P_{1}^{\prime}$. We put these neighborhoods of all the 1 dimensional affine patches in $\mathfrak{Q}_{1}$ together and denote this new set as $\mathfrak{Q}_{1}^{\prime}$. It is obvious that

$$
\mathfrak{P}_{1}^{\prime}=\mathfrak{Q}_{1}^{\prime} \cup \mathfrak{E}_{1}
$$


and $\mathfrak{P}_{1}$ have the same set of unit direction vectors of line segments on them.

Let us consider $\mathfrak{P}_{2}$. Similarly to $\mathfrak{P}_{1}$, we first decompose $\mathfrak{P}_{2}$ to be

$$
\mathfrak{P}_{2}=\left(\mathfrak{P}_{2} \backslash \mathfrak{E}_{2}\right) \cup \mathfrak{E}_{2} \triangleq \mathfrak{Q}_{2} \cup \mathfrak{E}_{2}
$$

From Assumption 5, for any $P_{2} \in \mathfrak{Q}_{2}$, there exists a closed 2D neighborhood $P_{2}^{\prime} \subset P_{2}$ (with positive 2D measure) such that no intersection between $P_{2}$ and the closure of the union of all the other affine patches in $\mathfrak{P}$ lies in this neighborhood. Again we collect all of these neighborhoods of all the affine patches in $\mathfrak{Q}_{2}$ and denote the new set as $\mathfrak{Q}_{2}^{\prime}$. We now generate a new set as follows:

$$
\mathfrak{P}_{2}^{\prime}=\mathfrak{Q}_{2}^{\prime} \cup \mathfrak{E}_{2} \text {. }
$$

For $\mathfrak{P}_{d}, d=3,4, \cdots, n-1$, with the decompositions

$$
\mathfrak{P}_{d}=\left(\mathfrak{P}_{d} \backslash \mathfrak{E}_{d}\right) \cup \mathfrak{E}_{d} \triangleq \mathfrak{Q}_{d} \cup \mathfrak{E}_{d}, d=3,4, \cdots, n-1,
$$

we apply similar procedures as above and get new sets $\mathfrak{Q}_{d}^{\prime}, \mathfrak{P}_{d}^{\prime}, d=3,4, \cdots, n-1$, with the following decompositions:

$$
\mathfrak{P}_{d}^{\prime}=\mathfrak{Q}_{d}^{\prime} \cup \mathfrak{E}_{d}, \quad d=3,4, \cdots, n-1 .
$$

We now consider the sets $\mathfrak{E}_{d}, d=1,2, \cdots, n-1$. Recall that

$$
\mathfrak{E}_{d}=\left\{P_{d}: P_{d} \in \mathfrak{P}_{d}, P_{d} \cap \mathrm{cl}\left(\cup_{P \in \mathfrak{P} \backslash\left\{P_{d}\right\}} P\right) \text { are dense on } P_{d}\right\}, d=1,2, \cdots, n-1 .
$$

We can now modify the affine patches in the sets $\mathfrak{E}_{d}, d=1,2, \cdots, n-1$. We start from $\mathfrak{E}_{n-1}$. Since any affine patch in $\bigcup_{1 \leq d \leq n-1} \mathfrak{Q}_{d}^{\prime}$ does not intersect with any affine patch in $\bigcup_{1 \leq d \leq n-1} \mathfrak{E}_{d}$, the only affine patches intersecting with $P_{n-1} \in \mathfrak{E}_{n-1}$ are in $\bigcup_{1 \leq d \leq n-1} \mathfrak{E}_{d}$, which are finitely many affine patches with lower or the same dimensions. We conclude that, for any affine patch $P_{n-1} \in \mathfrak{E}_{n-1}$, there exists a closed $n-1$ dimensional neighborhood $P_{n-1}^{\prime}$ such that no intersection between $P_{n-1}$ and affine patches in $\bigcup_{1 \leq d \leq n-1} \mathfrak{E}_{d}$ lies in this neighborhood. We collect all of these neighborhoods of all the affine patches in $\mathfrak{E}_{n-1}$ together and denote the new set as $\mathfrak{E}_{n-1}^{\prime}$. Similar procedures can be applied recursively to $\mathfrak{E}_{d}, d=n-2, \cdots, 1$, to generate $\mathfrak{E}_{d}^{\prime}, d=n-2, \cdots, 1$.

By the above procedures, we can construct $\tilde{\mathfrak{P}}$ as follows:

$$
\begin{aligned}
\tilde{\mathfrak{P}} & =\left(\mathfrak{Q}_{n-1}^{\prime} \cup \mathfrak{E}_{n-1}^{\prime}\right) \cup\left(\mathfrak{Q}_{n-2}^{\prime} \cup \mathfrak{E}_{n-2}^{\prime}\right) \cup \cdots \cup\left(\mathfrak{Q}_{1}^{\prime} \cup \mathfrak{E}_{1}^{\prime}\right) \\
& \triangleq \tilde{\mathfrak{P}}_{n-1} \cup \tilde{\mathfrak{P}}_{n-2} \cup \cdots \cup \tilde{\mathfrak{P}}_{1} .
\end{aligned}
$$

Note that the line segments in the affine patches in $\tilde{\mathfrak{P}}$ still have the same unit direction vectors as those in $Z$, i.e., $Z=\mathbf{v}(\tilde{\mathfrak{P}})$. However, the structure of $\tilde{\mathfrak{P}}$ is better for the following analysis. We have $\forall P, Q \in \tilde{\mathfrak{P}}, P \neq Q, P \cap Q=\emptyset$.

We now define the distance between two elements in $\tilde{\mathfrak{P}}$. For any $P, Q \in \tilde{\mathfrak{P}}$,

$$
\begin{aligned}
\operatorname{dist}(P, Q) & =\inf _{p \in P, q \in Q}\|p-q\|_{\mathbf{R}^{n}} \\
& =\min _{p \in P, q \in Q}\|p-q\|_{\mathbf{R}^{n}},
\end{aligned}
$$

where $\|p-q\|_{\mathbf{R}^{n}}$ is the usual Euclidean distance, since $P, Q$ are both closed sets. Then we have

$$
\forall P, Q \in \tilde{\mathfrak{P}}, P \neq Q, \operatorname{dist}(P, Q)>0
$$


Since $\mathfrak{E}_{d}^{\prime}, d=1,2, \cdots, n-1$ are all finite sets, there exists a natural number $k_{0}$ such that $\min _{P, Q \in \cup_{1 \leq d \leq n-1} \mathfrak{E}_{d}^{\prime}} \operatorname{dist}(P, Q)>\frac{1}{k_{0}}$. We define $\cup_{1 \leq d \leq n-1} \mathfrak{E}_{d}^{\prime}$ as $\tilde{\mathfrak{P}}^{k_{0}}$, that is, the set of all the affine patches (closed neighborhoods with various dimensions) in $\cup_{1 \leq d \leq n-1} \mathfrak{E}_{d}^{\prime}$. From $\tilde{\mathfrak{P}}^{k_{0}}$, we construct a sequence $\tilde{\mathfrak{P}}^{k}, k=k_{0}, k_{0}+1, \cdots$ as follows. Starting from $k=k_{0}$, for any $k$ and accordingly $\tilde{\mathfrak{P}}^{k}$, there exists a superset (maybe multiple sets, but we choose only one) in which any two elements have distance $>\frac{1}{k+1}$ and this property will become false if any new element is added. This superset is denoted as $\tilde{\mathfrak{P}}^{k+1}$. We thus obtain a sequence $\left\{\tilde{\mathfrak{P}}^{k}, k=k_{0}, k_{0}+1, \cdots\right\}$ satisfying

- $\forall P, Q \in \tilde{\mathfrak{P}}^{k}, \operatorname{dist}(P, Q)>\frac{1}{k}, k=k_{0}, k_{0}+1, \cdots$;

- $\forall P \in \tilde{\mathfrak{P}} \backslash \tilde{\mathfrak{P}}^{k}, \exists Q \in \tilde{\mathfrak{P}}^{k}$, s.t. $\operatorname{dist}(P, Q) \leq \frac{1}{k}, k=k_{0}+1, k_{0}+2, \cdots$.

By taking into account that any element of $\tilde{\mathfrak{P}}$ is a closed set and $\tilde{\mathfrak{P}}^{k_{0}}=\cup_{1 \leq d \leq n-1} \mathfrak{E}_{d}^{\prime}$, it can be verified by contradiction that

$$
\tilde{\mathfrak{P}}=\bigcup_{k=k_{0}}^{\infty} \tilde{\mathfrak{P}}^{k}
$$

According to the construction of $\tilde{\mathfrak{P}}^{k}$, for any $P \in \tilde{\mathfrak{P}}^{k}$, we can assign a neighborhood (surface patch) $B_{P} \subset B$ with positive $n-1$ dimensional surface area $A\left(B_{P}\right)$ such that $P \subset B_{P}$ and $\forall P, Q \in \tilde{\mathfrak{P}}^{k}, B_{P} \cap B_{Q}=\emptyset$. We have

$$
\tilde{\mathfrak{P}}^{k}=\bigcup_{j=1}^{\infty}\left\{P \in \tilde{\mathfrak{P}}^{k}: A\left(B_{P}\right)>\frac{1}{j}\right\} \triangleq \bigcup_{j=1}^{\infty} \tilde{\mathfrak{P}}^{k, j} .
$$

As above, the set of all the unit direction vectors of line segments in affine patches in $\tilde{\mathfrak{P}}^{k}$ is denoted as $\mathbf{v}\left(\tilde{\mathfrak{P}}^{k}\right)$; the set of all the unit direction vectors of line segments in affine patches in $\tilde{\mathfrak{P}}^{k, j}$ is denoted as $\mathbf{v}\left(\tilde{\mathfrak{P}}^{k, j}\right)$. We further denote

$$
\begin{aligned}
Y^{k}=\left\{\mathbf{v}_{n-1}\right. & =\left(v_{1}, v_{2}, \cdots, v_{n-1}\right) \in \mathbf{R}^{n-1}: \\
\mathbf{v} & \left.=\left(v_{1}, v_{2}, \cdots, v_{n-1}, \sqrt{1-\sum_{1 \leq i \leq n-1} v_{i}^{2}}\right) \in \mathbf{v}\left(\tilde{\mathfrak{P}}^{k}\right)\right\},
\end{aligned}
$$

and

$$
\begin{aligned}
Y^{k, j}=\left\{\mathbf{v}_{n-1}\right. & =\left(v_{1}, v_{2}, \cdots, v_{n-1}\right) \in \mathbf{R}^{n-1}: \\
\mathbf{v} & \left.=\left(v_{1}, v_{2}, \cdots, v_{n-1}, \sqrt{1-\sum_{1 \leq i \leq n-1} v_{i}^{2}}\right) \in \mathbf{v}\left(\tilde{\mathfrak{P}}^{k, j}\right)\right\} .
\end{aligned}
$$

We have

$$
Y^{k}=\bigcup_{j=1}^{\infty} Y^{k, j}
$$

For any fixed $k, j$, due to the finite total area of the boundary $B, \tilde{\mathfrak{P}}^{k, j}$ contains only finitely many affine patches. Therefore, $Y^{k, j}$ contains nothing but the union of finitely many $d$ dimensional circles with $d \in\{n-2, \cdots, 1\}$ in the unit $n-1$ dimensional ball. This indicates that $Y^{k, j}$ is measurable and has zero measure in $\mathbf{R}^{n-1}$. Hence, $Y^{k}$ is measurable and has zero measure in $\mathbf{R}^{n-1}$. We further have that the following set

$$
Y=\left\{\mathbf{v}_{n-1}=\left(v_{1}, v_{2}, \cdots, v_{n-1}\right) \in \mathbf{R}^{n-1}:\right.
$$




$$
\left.\mathbf{v}=\left(v_{1}, v_{2}, \cdots, v_{n-1}, \sqrt{1-\sum_{1 \leq i \leq n-1} v_{i}^{2}}\right) \in \mathbf{v}(\tilde{\mathfrak{P}})\right\}
$$

which is actually

$$
\begin{aligned}
Y=\left\{\mathbf{v}_{n-1}\right. & =\left(v_{1}, v_{2}, \cdots, v_{n-1}\right) \in \mathbf{R}^{n-1}: \\
\mathbf{v} & \left.=\left(v_{1}, v_{2}, \cdots, v_{n-1}, \sqrt{1-\sum_{1 \leq i \leq n-1} v_{i}^{2}}\right) \in Z\right\},
\end{aligned}
$$

is measurable and has measure zero in $\mathbf{R}^{n-1}$, since $Y=\bigcup_{k=k_{0}}^{\infty} Y^{k}$.

We now decompose $Y$ as

$$
Y=Y_{0} \cup Y_{+},
$$

where

$$
Y_{0}=\left\{\mathbf{v}_{n-1}=\left(v_{1}, v_{2}, \cdots, v_{n-1}\right) \in \mathbf{R}^{n-1}: \mathbf{v}=\left(v_{1}, v_{2}, \cdots, v_{n-1}, 0\right) \in Z\right\},
$$

and

$$
\begin{aligned}
Y_{+}=\left\{\mathbf{v}_{n-1}\right. & =\left(v_{1}, v_{2}, \cdots, v_{n-1}\right) \in \mathbf{R}^{n-1}:\left\|\mathbf{v}_{n-1}\right\|_{\mathbf{R}^{n-1}}<1, \\
\mathbf{v} & \left.=\left(v_{1}, v_{2}, \cdots, v_{n-1}, \sqrt{1-\sum_{1 \leq i \leq n-1} v_{i}^{2}}\right) \in Z\right\},
\end{aligned}
$$

Accordingly, we have

$$
Z=Z_{0} \cup Z_{+}
$$

where

$$
Z_{0}=\left\{\mathbf{v}=\left(v_{1}, v_{2}, \cdots, v_{n}\right) \in S_{\frac{1}{2}}^{n-1}: v_{n}=0, \mathbf{v}_{n-1}=\left(v_{1}, v_{2}, \cdots, v_{n-1}\right) \in Y_{0}\right\}
$$

and

$$
\begin{aligned}
Z_{+} & =\left\{\mathbf{v}=\left(v_{1}, v_{2}, \cdots, v_{n}\right) \in S_{\frac{1}{2}}^{n-1}: v_{n}>0, \mathbf{v}_{n-1}=\left(v_{1}, v_{2}, \cdots, v_{n-1}\right) \in Y_{+}\right\} \\
& =\left\{\mathbf{v}=\left(v_{1}, v_{2}, \cdots, v_{n}\right) \in S_{+}^{n-1}: \mathbf{v}_{n-1}=\left(v_{1}, v_{2}, \cdots, v_{n-1}\right) \in Y_{+}\right\}
\end{aligned}
$$

As a subset of $\left\{\mathbf{v}=\left(v_{1}, v_{2}, \cdots, v_{n}\right) \in S^{n-1}: v_{n}=0\right\}, Z_{0}$ has zero spherical measure. As a subset of $Y, Y_{+}$has zero measure in $\mathbf{R}^{n-1}$. On the other hand, $Y_{+}$can be reformulated as

$$
Y_{+}=\bigcup_{k=1}^{\infty}\left\{\mathbf{v}_{n-1} \in Y_{+}: v_{n}=\sqrt{1-\sum_{1 \leq i \leq n-1} v_{i}^{2}}>\frac{1}{k}\right\}
$$

By Lemma 3.1, one can show that $Z_{+}$has zero spherical measure. The theorem is proven.

THEOREM 3.3. In transmission imaging with parallel line geometry, for almost every projection direction $\mathbf{v} \in S_{\frac{1}{2}}^{n-1}$, the image taken in direction $\mathbf{v}$ is a continuous function. 
The subset of projection directions generating discontinuous images has zero measure on $S_{\frac{1}{2}}^{n-1}$.

Proof. We will prove that $\forall \mathbf{v} \in S_{\frac{1}{2}}^{n-1} \backslash Z, I_{\mathbf{v}}^{t}$ is a continuous function. Here $Z$ is as in Theorem 3.2.

According to our assumptions, $\forall \mathbf{v} \in S_{\frac{1}{2}}^{n-1} \backslash Z$, any straight line with direction $\mathbf{v}$ intersects with $B$ at at most finitely many points.

Without loss of generality, we can assume $\mathbf{v}=(0,0, \cdots, 0,1)$ (if $(0,0, \cdots, 0,1) \in Z$, we can rotate the coordinate system to make sure in the new system the $n^{\prime}$ th axis is not in $Z)$. In this case, we will generate an image whose pixels are in the $\mathbf{R}^{n-1}$ space with coordinates $\mathbf{r}_{n-1}=\left(r_{1}, r_{2}, \cdots, r_{n-1}\right)$. We denote this image as $I_{\mathbf{v}}^{t}\left(o ; r_{1}, r_{2}, \cdots, r_{n-1}\right)$. We now show that it is a continuous function with respect to the image coordinates $\left(r_{1}, r_{2}, \cdots, r_{n-1}\right)$. At any $\mathbf{r}_{n-1}^{*}=\left(r_{1}^{*}, r_{2}^{*}, \cdots, r_{n-1}^{*}\right) \in \mathbf{R}^{n-1}$, the straight line for integration can be written as

$$
\mathbf{r}=\left(\mathbf{r}_{n-1}^{*}, 0\right)+\mathbf{v} t,-\infty<t<+\infty,
$$

which is, in short, $l_{\left(\mathbf{r}_{n-1}^{*}, 0\right), \mathbf{v}}$. We discuss the continuity in three cases as follows.

a. $l_{\left(\mathbf{r}_{n-1}^{*}, 0\right), \mathbf{v}} \cap B=\emptyset$. In this case, there exists a neighborhood $B_{\mathbf{r}_{n-1}^{*}, \delta} \subset \mathbf{R}^{n-1}$, such that $\forall \mathbf{r}_{n-1} \in B_{\mathbf{r}_{n-1}^{*}, \delta}, l_{\left(\mathbf{r}_{n-1}, 0\right), \mathbf{v}} \cap B=\emptyset$. Therefore, the image intensity $I_{\mathbf{v}}^{t}\left(o ; \mathbf{r}_{n-1}\right)=0$ for $\forall \mathbf{r}_{n-1} \in B_{\mathbf{r}_{n-1}^{*}, \delta}$. It is thus continuous at $\mathbf{r}_{n-1}^{*}$.

b. $l_{\left(\mathbf{r}_{n-1}^{*}, 0\right), \mathbf{v}} \cap B=\left\{\left(\mathbf{r}_{n-1}^{*}, r_{n}^{*, i}\right): i=1, \cdots, 2 M, r_{n}^{*, 1}<r_{n}^{*, 2}<\cdots<r_{n}^{*, 2 M}\right\}$ and all the intersecting points are secant points. By the continuity of the boundary $B$ and that any intersecting point is a secant point, $\forall 1 \leq i \leq 2 M$, there exists a local continuous mapping $\varphi^{i}$ from a small neighborhood $B_{\mathbf{r}_{n-1}^{*}, \delta^{i}} \subset \mathbf{R}^{n-1}$ to a small neighborhood of $\left(\mathbf{r}_{n-1}^{*}, r_{n}^{*, i}\right)$ on $B$, which is

$$
\varphi^{i}\left(\mathbf{r}_{n-1}\right)=\left(\mathbf{r}_{n-1}, h^{i}\left(\mathbf{r}_{n-1}\right)\right), \forall \mathbf{r}_{n-1} \in B_{\mathbf{r}_{n-1}^{*}, \delta^{i}},
$$

for some continuous function $h^{i}\left(\mathbf{r}_{n-1}\right)$ (otherwise there will be more than one intersection between $l_{\left(\mathbf{r}_{n-1}^{*}, 0\right), \mathbf{v}}$ and $B$ around this secant point). This also means that, $\forall 1 \leq i \leq 2 M$, the secant point $\left(\mathbf{r}_{n-1}^{*}, r_{n}^{*, i}\right)$ is in the interior of the set $\varphi^{i}\left(B_{\mathbf{r}_{n-1}^{*}, \delta^{i}}\right)$. It follows that, $\forall \mathbf{r}_{n-1} \in B_{\mathbf{r}_{n-1}^{*}, \delta^{i}}, l_{\left(\mathbf{r}_{n-1}, 0\right), \mathbf{v}}$ intersects with $\varphi^{i}\left(B_{\mathbf{r}_{n-1}^{*}, \delta^{i}}\right)$ at a secant point. Note that we can choose $\delta^{i}$ small enough so that $\varphi^{i}\left(B_{\mathbf{r}_{n-1}^{*}, \delta^{i}}\right) \cap \varphi^{j}\left(B_{\mathbf{r}_{n-1}^{*}, \delta^{j}}\right)=\emptyset, \forall i \neq j$, since $r_{n}^{*, 1}<r_{n}^{*, 2}<\cdots<r_{n}^{*, 2 M}$. Let $\delta=\min \left(\delta^{1}, \delta^{2}, \cdots, \delta^{2 M}\right)$. We conclude that, $\forall \mathbf{r}_{n-1} \in B_{\mathbf{r}_{n-1}^{*}, \delta}, l_{\left(\mathbf{r}_{n-1}, 0\right), \mathbf{v}}$ intersects with $B$ at a series of secant points $\left\{\left(\mathbf{r}_{n-1}, r_{n}^{i}\right): 1 \leq i \leq 2 M, r_{n}^{1}<r_{n}^{2}<\right.$ $\left.\cdots<r_{n}^{2 M}\right\}$. Again by the continuity, $\forall i=1,2, \cdots, 2 M,\left|r_{n}^{i}-r_{n}^{*, i}\right|<\hat{\delta}$. and $\hat{\delta} \rightarrow 0$ as $\delta \rightarrow 0$. This, together with the continuity of the density function $\rho(\mathbf{r})$, indicates that

$$
\begin{aligned}
& I_{\mathbf{v}}^{t}\left(o ; \mathbf{r}_{n-1}\right)-I_{\mathbf{v}}^{t}\left(o ; \mathbf{r}_{n-1}^{*}\right) \\
= & \int_{l_{\left(\mathbf{r}_{n-1}, 0\right), \mathbf{v}}} \rho(\mathbf{r}) d l-\int_{l_{\left(\mathbf{r}_{n-1}^{*}, 0\right), \mathbf{v}}} \rho(\mathbf{r}) d l \\
= & \sum_{1 \leq i \leq M} \int_{r_{n}^{2 i-1}}^{r_{n}^{2 i}} \rho\left(\left(\mathbf{r}_{n-1}, t\right)\right) d t-\sum_{1 \leq i \leq M} \int_{r_{n}^{*}, 2 i-1}^{r_{n}^{*, 2 i}} \rho\left(\left(\mathbf{r}_{n-1}^{*}, t\right)\right) d t
\end{aligned}
$$




$$
\begin{aligned}
& =\sum_{\substack{1 \leq i \leq M\\
\\
}}\left(\int_{r_{n}^{2 i-1}}^{r_{n}^{2 i}} \rho\left(\left(\mathbf{r}_{n-1}, t\right)\right) d t-\int_{r_{n}^{*, 2 i-1}}^{r_{n}^{*, 2 i}} \rho\left(\left(\mathbf{r}_{n-1}^{*}, t\right)\right) d t\right) \\
&
\end{aligned}
$$

as $\delta \rightarrow 0$. Hence the image is continuous at $\mathbf{r}_{n-1}^{*}$.

c. $l_{\left(\mathbf{r}_{n-1}^{*}, 0\right), \mathbf{v}} \cap B=\left\{\left(\mathbf{r}_{n-1}^{*}, r_{n}^{*, i}\right): i=1, \cdots, 2 M+K, r_{n}^{*, 1}<r_{n}^{*, 2}<\cdots<r_{n}^{*, 2 M+K}\right\}$, within which $2 M$ points are secant points and the remainder are touching points. Assume that the index set of the secant points is $A_{1}$ whereas the index set of the touching points is $A_{2}$. First, let us consider the secant points. By the argument in the above case, there always exists a neighborhood $B_{\mathbf{r}_{n-1}^{*}, \delta_{1}}$ (with $\delta_{1}$ small enough) such that $\forall \mathbf{r}_{n-1} \in B_{\mathbf{r}_{n-1}^{*}, \delta_{1}}$ and $\forall i \in A_{1}$, $l_{\left(\mathbf{r}_{n-1}, 0\right), \mathbf{v}}$ intersects with $B$ around the secant point $\left(\mathbf{r}_{n-1}^{*}, r_{n}^{*, i}\right)$ at another secant point $\left(\mathbf{r}_{n-1}, r_{n}^{i}\right)$. We have $r_{n}^{i_{1}}<r_{n}^{i_{2}}, \forall i_{1}, i_{2} \in A_{1}, i_{1}<i_{2}$. In addition, by the continuity, $\forall i \in A_{1},\left|r_{n}^{i}-r_{n}^{*, i}\right|<\hat{\delta_{1}}$, and $\hat{\delta_{1}} \rightarrow 0$ as $\delta_{1} \rightarrow 0$.

Now let us consider the touching points. $\forall i \in A_{2}$, we decompose a small enough neighborhood of $\left(\mathbf{r}_{n-1}^{*}, r_{n}^{*, i}\right)$ on $B$ to the union of the upper half $U$ with the $n$th coordinate $r_{n} \geq r_{n}^{*, i}$ and the lower half $L$ with the $n$th coordinate $r_{n} \leq r_{n}^{*, i}$. There exists a continuous mapping from a subset of a neighborhood $B_{\mathbf{r}_{n-1}^{*}, \delta_{2}^{i,+}}$ to $U$, and a continuous mapping from a subset of a neighborhood $B_{\mathbf{r}_{n-1}^{*}, \delta_{2}^{i,-}}$ to $L$, respectively. Let $\delta_{2}^{i}=\min \left(\delta_{2}^{i,+}, \delta_{2}^{i,-}\right)$. Then, $\forall \mathbf{r}_{n-1} \in B_{\mathbf{r}_{n-1}^{*}, \delta_{2}^{i}}$, the straight line $l_{\left(\mathbf{r}_{n-1}, 0\right), \mathbf{v}}$ intersects $U \cup L$ at an empty set, or a single touching point $r_{n}^{i}$, or two secant points $r_{n}^{i,+}, r_{n}^{i,-}$. Again, by the continuity of the mappings, $\left|r_{n}^{i,+}-r_{n}^{*, i}\right|<\hat{\delta_{2}^{i}},\left|r_{n}^{i,-}-r_{n}^{*, i}\right|<\hat{\delta}_{2}^{i}$, and $\hat{\delta_{2}^{i}} \rightarrow 0$ as $\delta_{2}^{i} \rightarrow 0$. Now we let $\delta_{2}=\min \left\{\delta_{2}^{i}: i \in A_{2}\right\}$. Therefore, $\forall \mathbf{r}_{n-1} \in B_{\mathbf{r}_{n-1}^{*}, \delta_{2}}$ and $\forall i \in A_{2}$, the straight line $l_{\left(\mathbf{r}_{n-1}, 0\right), \mathbf{v}}$ intersects $B$ around the touching point $\left(\mathbf{r}_{n-1}^{*}, r_{n}^{*, i}\right)$ at an empty set, or a single point, or two secant points $r_{n}^{i,+}, r_{n}^{i,-}$. In addition, $\left|r_{n}^{i,+}-r_{n}^{*, i}\right|<\hat{\delta_{2}},\left|r_{n}^{i,-}-r_{n}^{*, i}\right|<\hat{\delta_{2}}$, and $\hat{\delta_{2}} \rightarrow 0$ as $\delta_{2} \rightarrow 0$.

Let $\delta=\min \left(\delta_{1}, \delta_{2}\right)$. We conclude that, $\forall \mathbf{r}_{n-1} \in B_{\mathbf{r}_{n-1}^{*}, \delta}$,

$$
I_{\mathbf{v}}^{t}\left(o ; \mathbf{r}_{n-1}\right)-I_{\mathbf{v}}^{t}\left(o ; \mathbf{r}_{n-1}^{*}\right) \rightarrow 0, \text { as } \delta \rightarrow 0,
$$

by a similar argument as in the previous case, with a trick to delete some of the touching points $\left\{\left(\mathbf{r}_{n-1}^{*}, r_{n}^{*, i}\right): i \in A_{2}\right\}$ and make the remainder of multiplicity 2 according to how many intersections there are between the line and $B$ around the touching points. The image is also continuous at $\mathbf{r}_{n-1}^{*}$ in this case.

Theorem 3.2 and 3.3 show that in transmission imaging with parallel line geometry, images taken in almost all the projection directions are continuous functions. The discontinuous images are so sparse that in real applications they seldomly appear. This will provide some information for processing images generated by transmission imaging, which is totally different from reflection imaging.

The following corollary is on our results applied to a combination of finite objects.

Corollary 3.4. Suppose that a physical scene consists of finite objects $\left\{o_{i}: 1 \leq i \leq\right.$ $M\}$ with compactly supported density functions $\left\{\rho_{i}(\mathbf{r}): 1 \leq i \leq M\right\}$ which are continuous over their supports $\left\{D_{i}: 1 \leq i \leq M\right\}$, respectively. The set of projection directions generating discontinuous images has measure zero on $S_{\frac{1}{2}}^{n-1}$. In particular, even if 
$\left\{\rho_{i}(\mathbf{r}) \equiv c_{i}, \mathbf{r} \in D_{i}: c_{i} \in \mathbf{R}\right.$ is a constant, $\left.1 \leq i \leq M\right\}$, i.e., the whole density is piecewise constant, the result still holds.

Since in real applications the case of $\mathbf{R}^{3}$ is very important, we give the following corollary.

Corollary 3.5. Assume that a physical scene in $\mathbf{R}^{3}$ consists of finite objects with compactly supported density functions which are continuous over their domains. The set of projection directions generating discontinuous images has measure zero on $S_{\frac{1}{2}}^{2}$.

3.2. Radial density functions. In some applications, the density functions are not compactly supported. However, they are radial functions or combinations of some translations of radial functions. This subsection contributes to this case.

Again we need only consider one single object. We denote it by $o$ and assume the density function is $\rho(|\mathbf{r}|), \mathbf{r} \in \mathbf{R}^{n}$. Since $\rho$ depends only on $|\mathbf{r}|$, the images are independent of projection directions. All the projection directions will generate the same image. Therefore, without loss of generality, we can assume the projection direction is $\mathbf{v}=(0,0, \cdots, 0,1)$, for convenience of description. The coordinates in the image plane is thus $\mathbf{r}_{n-1}$. We then have

$$
I_{\mathbf{v}}^{t}\left(o ; \mathbf{r}_{n-1}\right)=\int_{l_{\left(\mathbf{r}_{n-1}, 0\right), \mathbf{v}}} \rho(|\mathbf{r}|) d l=\int_{-\infty}^{+\infty} \rho\left(\sqrt{\left|\mathbf{r}_{n-1}\right|^{2}+r_{n}^{2}}\right) d r_{n} .
$$

It is straightforward to verify that the image $I_{\mathbf{v}}^{t}\left(o ; \mathbf{r}_{n-1}\right)$ is a radial function of $\mathbf{r}_{n-1}$. Under some mild assumptions, it is continuous.

TheOREM 3.6. The image $I_{\mathbf{v}}^{t}\left(o ; \mathbf{r}_{n-1}\right)$ is also radial, when the density of the object is a radial function.

TheOREM 3.7. Assume that $\forall \mathbf{r}_{n-1} \in \mathbf{R}^{n-1}, \int_{-\infty}^{+\infty} \rho^{2}\left(\sqrt{\left|\mathbf{r}_{n-1}\right|^{2}+r_{n}^{2}}\right) d r_{n}<+\infty$. Then the image $I_{\mathbf{v}}^{t}\left(o ; \mathbf{r}_{n-1}\right)$ is continuous with respect to $\mathbf{r}_{n-1}$.

Proof. Assume that $\mathbf{r}_{n-1}^{*} \in \mathbf{R}^{n-1}, \mathbf{r}_{n-1} \in \mathbf{R}^{n-1}$ and $\mathbf{r}_{n-1} \rightarrow \mathbf{r}_{n-1}^{*}$. Without loss of generality, we consider the case of $\left|\mathbf{r}_{n-1}\right|>\left|\mathbf{r}_{n-1}^{*}\right|$ (the case of $\left|\mathbf{r}_{n-1}\right|<\left|\mathbf{r}_{n-1}^{*}\right|$ can be treated similarly). Let $z=\sqrt{\left|\mathbf{r}_{n-1}\right|^{2}-\left|\mathbf{r}_{n-1}^{*}\right|^{2}}$. We have

$$
\begin{aligned}
I_{\mathbf{v}}^{t}\left(o ; \mathbf{r}_{n-1}\right)-I_{\mathbf{v}}^{t}\left(o ; \mathbf{r}_{n-1}^{*}\right)= & \int_{-\infty}^{+\infty} \rho\left(\sqrt{\left|\mathbf{r}_{n-1}\right|^{2}+r_{n}^{2}}\right) d r_{n}-\int_{-\infty}^{+\infty} \rho\left(\sqrt{\left|\mathbf{r}_{n-1}^{*}\right|^{2}+r_{n}^{2}}\right) d r_{n} \\
= & \int_{-\infty}^{+\infty} \rho\left(\sqrt{\left|\mathbf{r}_{n-1}\right|^{2}+r_{n}^{2}}\right) d r_{n} \\
& \quad-\left(\int_{-\infty}^{-z}+\int_{-z}^{z}+\int_{z}^{+\infty}\right) \rho\left(\sqrt{\left|\mathbf{r}_{n-1}^{*}\right|^{2}+r_{n}^{2}}\right) d r_{n} \\
= & 2 \int_{0}^{+\infty} \rho\left(\sqrt{\left|\mathbf{r}_{n-1}\right|^{2}+r_{n}^{2}}\right) d r_{n}-2 \int_{z}^{+\infty} \rho\left(\sqrt{\left|\mathbf{r}_{n-1}^{*}\right|^{2}+r_{n}^{2}}\right) d r_{n} \\
& -\int_{-z}^{z} \rho\left(\sqrt{\left|\mathbf{r}_{n-1}^{*}\right|^{2}+r_{n}^{2}}\right) d r_{n} \\
= & 2 \int_{0}^{+\infty} \rho\left(\sqrt{\left|\mathbf{r}_{n-1}\right|^{2}+r_{n}^{2}}\right)\left(1-\frac{r_{n}}{\sqrt{r_{n}^{2}+z^{2}}}\right) d r_{n} \\
& \quad-\int_{-z}^{z} \rho\left(\sqrt{\left|\mathbf{r}_{n-1}^{*}\right|^{2}+r_{n}^{2}}\right) d r_{n}
\end{aligned}
$$




$$
=\mathrm{I}_{1}+\mathrm{I}_{2} \text {. }
$$

We now estimate the integral $\mathrm{I}_{1}$.

$$
\begin{aligned}
\left|\mathrm{I}_{1}\right|^{2} & \leq 4 \int_{0}^{+\infty} \rho^{2}\left(\sqrt{\left|\mathbf{r}_{n-1}\right|^{2}+r_{n}^{2}}\right) d r_{n} \int_{0}^{+\infty}\left(1-\frac{r_{n}}{\sqrt{r_{n}^{2}+z^{2}}}\right)^{2} d r_{n} \\
& =4 \int_{0}^{+\infty} \rho^{2}\left(\sqrt{\left|\mathbf{r}_{n-1}\right|^{2}+r_{n}^{2}}\right) d r_{n} \int_{0}^{+\infty}\left(\frac{\sqrt{r_{n}^{2}+z^{2}}-r_{n}}{\sqrt{r_{n}^{2}+z^{2}}}\right)^{2} d r_{n} \\
& <4 \int_{0}^{+\infty} \rho^{2}\left(\sqrt{\left|\mathbf{r}_{n-1}\right|^{2}+r_{n}^{2}}\right) d r_{n} \int_{0}^{+\infty} \frac{z^{2}}{r_{n}^{2}+z^{2}} d r_{n} \\
& =\pi z \int_{-\infty}^{+\infty} \rho^{2}\left(\sqrt{\left|\mathbf{r}_{n-1}\right|^{2}+r_{n}^{2}}\right) d r_{n} .
\end{aligned}
$$

As for the integral $\mathrm{I}_{2}$, the absolute continuity of integral gives that $\left|\mathrm{I}_{2}\right| \rightarrow 0$, as $z \rightarrow 0$.

Therefore, as $\mathbf{r}_{n-1} \rightarrow \mathbf{r}_{n-1}^{*}$ and thus $z \rightarrow 0$, we have $\left|\mathrm{I}_{1}\right|+\left|\mathrm{I}_{2}\right| \rightarrow 0$, which implies $I_{\mathbf{v}}^{t}\left(o ; \mathbf{r}_{n-1}\right)$ is continuous at $\mathbf{r}_{n-1}^{*}$.

COROLlary 3.8. Suppose that a physical scene consisting of finite objects $\left\{o_{i}: 1 \leq\right.$ $i \leq M\}$ with translations of radial density functions $\left\{\rho_{i}\left(\left|\mathbf{r}-\mathbf{r}_{i}\right|\right), \mathbf{r}_{i} \in \mathbf{R}^{n}: 1 \leq i \leq M\right\}$ satisfying the condition in Theorem 3.7. Then $\forall \mathbf{v} \in S_{\frac{1}{2}}^{n-1}, I_{\mathbf{v}}^{t}\left(o_{1}+o_{2}+\cdots+o_{M} ; \cdot\right)$ is continuous.

\section{Improving TV regularization for Poisson noise removal}

In this section we present a straightforward application of our analysis, i.e., a simple yet effective improvement of the popular TV regularization for Poisson noise reduction of images generated by transmission imaging. We should mention that there are also many other very successful image denoising methods which are not based on energy minimization and the variational principle.

TV regularization was first introduced in [29] and has been extensively applied in various digital image processing problems; see [9] and references therein. The definition in 2D image processing is as follows:

$$
\operatorname{TV}(u)=\int_{\Omega}|\nabla u| d \Omega=\sup \left\{\int_{\Omega} u \operatorname{div} \xi d \Omega: \xi \in C_{c}^{1}\left(\Omega ; \mathbf{R}^{2}\right),\|\xi\|_{\infty} \leq 1\right\},
$$

where $u$ is a function defined on $\Omega \subset \mathbf{R}^{2}$; and $C_{c}^{1}\left(\Omega ; \mathbf{R}^{2}\right)$ is the space of $C^{1}$ mappings from $\Omega$ to $\mathbf{R}^{2}$ with compact supports. TV has many extensions such as multi-channel TV and high order models. Since high order models are much more related to our problem, we review these generalizations in the following. High order models were mainly proposed for the reduction of staircase effect of TV or for image decomposition and texture extraction. Most of them allow piecewise affine linear reconstructions or decompositions. Similarly to [2], we categorize them to be the following three classes. They are structurally quite different from each other, as one can see from their dual formulations.

- Generalizations by directly involving high order derivatives. This class has the following form:

$$
J(u)=\int_{\Omega} \phi\left(u, \nabla u, \nabla^{2} u, \cdots, \nabla^{k} u\right) d \Omega
$$


where $\phi$ is some positive function and possibly convex. Examples include $[7,17,21,26,30,35,41]$ where the models are defined via either primal or dual formulations.

- Generalizations by infimal convolution. This class has the following form:

$$
J(u)=\left(J_{1} \square \cdots \square J_{k}\right)(u)=\inf _{u=u_{1}+\cdots+u_{k}} \sum_{1 \leq i \leq k} J_{i}\left(u_{i}\right),
$$

where $J_{i}\left(u_{i}\right), 1 \leq i \leq k$ are some convex functionals which may involve high order derivatives such as Hessian and Laplacian of the their arguments. Examples include $[6,8,31,33]$, among which [33] is a general modification of infimal convolution based approach.

- Total generalized variation (TGV). This is a very new generalization of total variation regularization, which reads

$$
\begin{array}{r}
\operatorname{TGV}_{\alpha}^{k}(u)=\sup \left\{\int_{\Omega} u \operatorname{div}^{k} \xi d \Omega: \xi \in C_{c}^{k}\left(\Omega ; \operatorname{Sym}^{k}\left(\mathbf{R}^{2}\right)\right),\right. \\
\left.\left\|\operatorname{div}^{i} \xi\right\|_{\infty} \leq \alpha_{i}, 0 \leq i \leq k-1\right\},
\end{array}
$$

where $\operatorname{Sym}^{k}\left(\mathbf{R}^{2}\right)$ is the space of symmetric $k$-tensors and $\alpha$ is a parameter vector; see [3], [19] for more details. TGV has the ability to balance different orders of derivatives. We mention that the modified infimal convolution [33] is a discrete variant of TGV.

These high order generalizations achieved great successes in staircase reduction and image decomposition.

Very recently, many scholars studied the combination of TV and the KullbackLeibler (KL) fidelity [24, 20] for Poisson noise removal [4, 10, 20, 12, 24, 32, 40, 42] and many numerical tests therein used piecewise constant images. The minimization model reads

$$
\min _{u} \int_{\Omega}|\nabla u| d \Omega+\alpha \int_{\Omega}(u-f \log u) d \Omega,
$$

where $f$ is an observed image defined on $\Omega \subset \mathbf{R}^{2}$, and $\alpha$ is a model parameter. As in [40], we call this model as TV-KL model.

However, according to our results in the previous section, images generated by transmission imaging are usually continuous. Since TV often suffers from the staircase effect and generates piecewise constant solutions, it destroys the continuity structure of the true images by transmission imaging. Hence the TV-KL model needs to be improved.

Intuitively we would like to consider high order regularizations. This is our first try after realizing the continuity property of images by transmission imaging. We therefore propose to solve the following simple one:

$$
\min _{u} \int_{\Omega} \sqrt{u_{x}^{2}+u_{y}^{2}+u_{x x}^{2}+u_{x y}^{2}+u_{y x}^{2}+u_{y y}^{2}} d \Omega+\alpha \int_{\Omega}(u-f \log u) d \Omega,
$$

which combines the first order and the second order derivatives $\nabla u$ and Hessian $(u)$. We call (4.3) as MoTV(Multi-order TV)-KL model, since it uses a combination of $\nabla u$ and Hessian $(u)$. Here we give the following remarks to this model. 

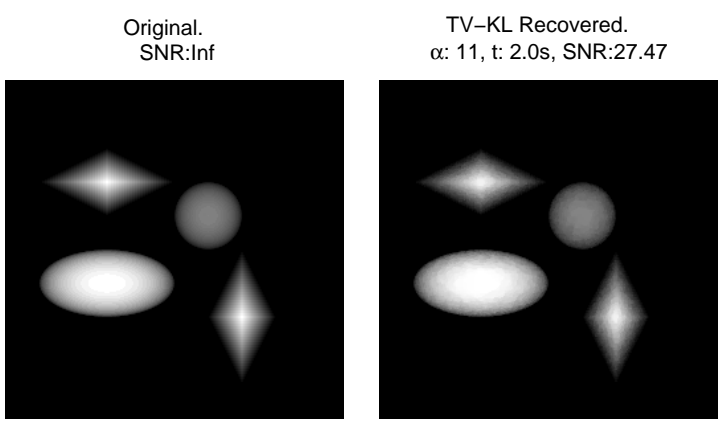

MoTV-KL Recovered.
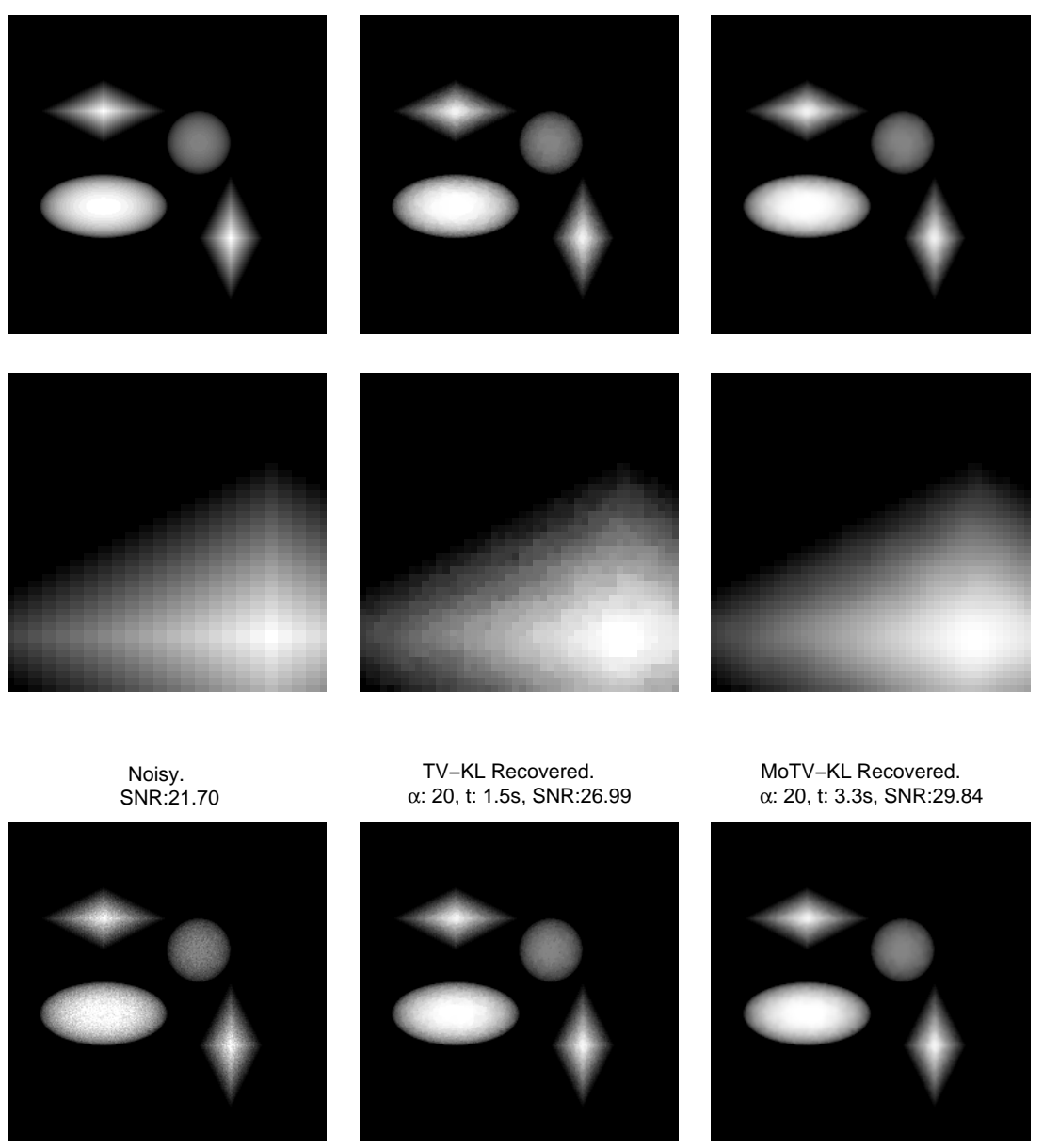

MoTV-KL Recovered. $\alpha: 20$, t: 3.3s, SNR:29.84
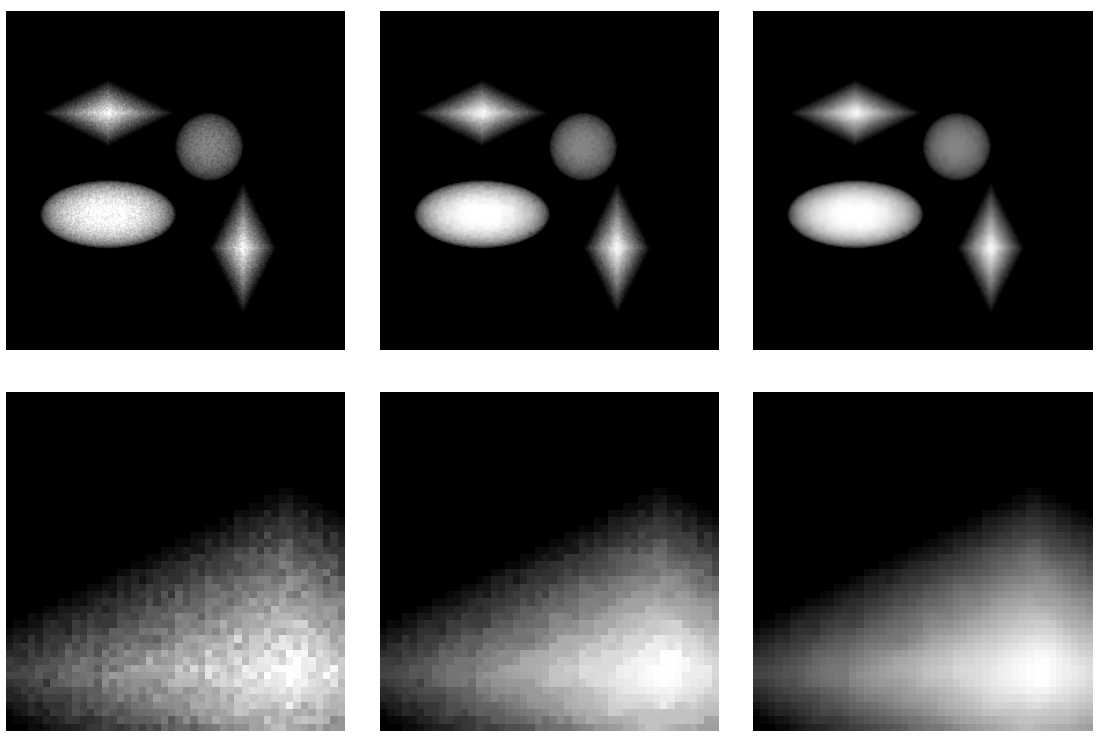

FIG. 4.1. Comparisons between the TV-KL and MoTV-KL models for a $256 \times 256$ synthetic image. The second and fourth rows are zoom-in images of the first and third rows, respectively. The first column: original and noisy (with Poisson noise) images. The second column: TV-KL recovered images. The third column: MoTV-KL recovered images. According to our test, the TV-KL model gives a result with nearly highest $S N R$ with the model parameter $\alpha=11$, whereas the MoTV-KL model generates a result with nearly highest SNR with $\alpha=20$. For both parameter values, the MoTV-KL model gives much higher SNR than the TV-KL model. In addition, the TV-KL model suffers from staircase effect, whereas the MoTV-KL model does not. 

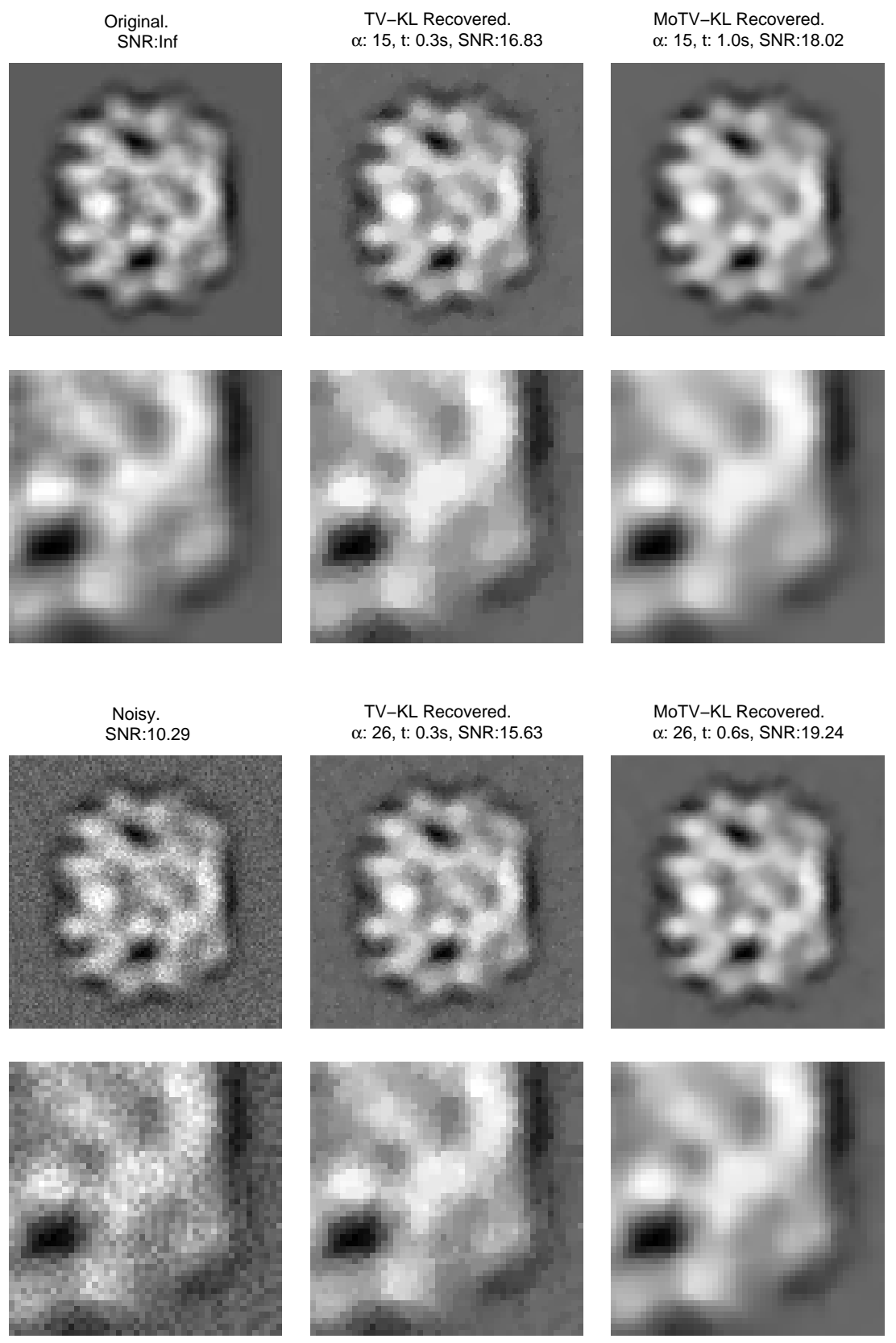

FIG. 4.2. Comparisons between the TV-KL and MoTV-KL models for a $95 \times 95$ molecular image. The second and fourth rows are zoom-in images of the first and third rows, respectively. The first column: original and noisy (with Poisson noise) images. The second column: TV-KL recovered images. The third column: MoTV-KL recovered images. According to our test, the TV-KL model gives a result with nearly highest $S N R$ with the parameter $\alpha=15$, whereas the MoTV-KL model gives a result with nearly highest $S N R$ with $\alpha=26$. For both parameters, the MoTV-KL model gives much higher SNR than the TV-KL model. In addition, the TV-KL model suffers from staircase effect, whereas the MoTV-KL model does not. 
REMARK 4.1. The regularizer in (4.3) is an extension of TV and not new. It falls in the first class of high order generalizations listed above. Here we just combine it with KL fidelity to remove Poisson noise.

REMARK 4.2. Since the underlying density functions of imaged objects are diverse, the corresponding most suitable regularizers should be also diverse. To the best of our understanding, the proposed MoTV-KL model (4.3) may be the simplest improvement of the TV-KL model. Although it seems to favor piecewise linear images, which are the simplest non-constant continuous images, it also at least improves the effectiveness of the TV-KL model for more complicated continuous images (our test images are not restricted to be piecewise linear images). This section is devoted not for a perfect denoising model for all continuous images, but a simple yet effective improvement of the TV-KL model, showing a straightforward application of our theoretical analysis.

REMARK 4.3. According to our test, the model involving only the second order derivatives Hessian $(u)$ does not work well. Therefore, the $\nabla u$ in (4.3) cannot be deleted. This indicates that, in denoising problems, $\nabla u$ in regularization is even quite important for images with no discontinuity. This also verifies Remark 4.2 in the sense that the involvement of Hessian $(u)$ in the MoTV-KL model is helpful for continuous images with continuity orders higher than piecewise linearity.

REMARK 4.4. The aim of this section is to show that TV regularization for Poisson noise removal can be greatly improved, verifying our theoretical analysis and giving a straightforward application of our continuity result. As our first try after realizing the continuity property, we therefore propose to solve a simple model, which still exhibits much improvement. This is the first high order variational model applied to Poisson noise removal. Of course many other high order methods could also be used, such as infimal convolution methods and TGV. We plan to report a detailed summary study on comparisons between several major high order methods and wavelet methods in our future.

The problem (4.3) is an $L_{1}$ minimization problem. Recently many efficient methods have been developed to solve this kind of problem; see, e.g., [5, 14, 36, 39, 40] and references therein. In our implementation we applied operator splitting and an augmented Lagrangian method with single inner iteration [13] to solve it based on our previous work [36, 39, 40].

Two numerical examples are shown in figures 4.1 and 4.2. In figure 4.1, the image is synthesized by projecting 4 objects ( 1 sphere +2 polyhedrons +1 ellipse, with constant density functions within their domains, respectively) to an image plane. The molecular image in figure 4.2 was downloaded from http://blake.bcm.edu/emanwiki/EMAN2. The experiments were performed under Windows Vista and Matlab R14 (Version 7.0.4) on a laptop with Intel CPU (Core 2) at $2.53 \mathrm{GHz}$ and $4 \mathrm{~GB}$ memory. We used $\frac{\left\|u^{k}-u^{k-1}\right\|_{F}}{\left\|u^{k-1}\right\|_{F}} \leq 10^{-4}$ as the stopping condition of the iteration for all the examples, where $\|\cdot\|_{F}$ denotes the Frobenius norm of the data expressed as a matrix. From figures 4.1 and 4.2 , one can clearly see the continuity of the clean images. The MoTV-KL model (4.3) generates much smoother results with much higher SNRs than the TV-KL model (4.2). In the tests, we adjusted the model parameter $\alpha$ to figure out how high SNRs the two models can achieve respectively. For the parameter value with which the TV-KL model generates nearly highest SNR, we computed the result by the MoTV-KL model, and vice versa. We found that 
the MoTV-KL model gives results with much higher SNRs, even with the parameter value which is best for the TV-KL model. Besides, the TV-KL model suffers from staircase effect, whereas the MoTV-KL model does not. The fact that the smoother solutions have higher SNRs also demonstrates our analysis on the continuity of the images generated by transmission imaging with parallel line geometry. 


\section{Conclusions and future work}

In this paper, we analyzed the continuity property of images generated by transmission imaging with parallel line geometry of wave beam (which is a fundamental and widely used geometry) and showed a simple yet natural application of our theoretical analysis. Transmission imaging is widely applied in astronomy and biomedical sciences for macro and micro scale objects, which is quite different from reflection imaging frequently used in our everyday life for common scale objects. As is well known, images generated by reflection imaging are usually modeled as discontinuous functions. However, we showed that almost all images generated by transmission imaging are continuous functions, even if the density functions of the imaged objects are discontinuous. Discontinuous images scarcely appear in real transmission imaging applications. This analysis has not appeared yet in the literature. Our results may help us to understand the structures of images generated by transmission imaging and provide some information for designing and testing image processing techniques for transmission imaging. As a straightforward application, we proposed a simple yet effective improvement of the most popular TV regularization applied to Poisson noise removal. In addition, more reasonable test images for models and algorithms should be continuous, instead of the currently widely used piecewise constant images and other discontinuous images. Numerical tests and comparisons demonstrated our analysis and the effectiveness of the improved variational model.

There are some future works. In this paper we only considered the transmission imaging with parallel line geometry. The corresponding results for fan beam geometry will be reported in our near future. In addition, the variational model presented here is just our first try to handle this kind of images after our realizing the continuity of the images. We believe that better restoration models exist and need to be found. Since the density functions of the imaged objects are diverse, ideally we would like to construct good models which can adaptively capture the local structures of the images. This is not easy. We will first test and compare several major high order methods and wavelet methods.

Acknowledgment. We thank Prof. Weixiao Shen and Prof. Zuowei Shen for their helpful discussions and suggestions. We also thank the National Center for Macromolecular Imaging (http://ncmi.bcm.edu/ncmi) for providing the second test image online.

\section{REFERENCES}

[1] G. Aubert and P. Kornprobst, Mathematical Problems in Image Processing: Partial Differential Equations and the Calculus of Variations, second edition, Series of Applied Mathematical Sciences, Springer-Verlag, 147, 2006.

[2] M. Benning, C. Brune, M. Burger, and J. Muller, Higher-Order TV Methods - Enhancement via Bregman Iteration, UCLA CAM Report, 12-04, 2012.

[3] K. Bredies, K. Kunisch, and T. Pock, Total generalized variation, SIAM J. Imaging Sci., 3(3), 492-526, 2010.

[4] C. Brune, A. Sawatzky, and M. Burger, Bregman-EM-TV methods with application to optical nanoscopy, LNCS, 5567, 235-246, 2009.

[5] J. Cai, S. Osher, and Z. Shen, Split Bregman methods and frame based image restoration, Multiscale Model. Simul., 8, 337-369, 2009.

[6] A. Chambolle and P.L. Lions, Image recovery via total variation minimization and related problems, Numer. Math., 76, 167-188, 1997.

[7] T. Chan, A. Marquina, and P. Mulet, High-order total variation-based image restoration, SIAM J. Sci. Comput., 22, 503-516, 2000.

[8] T.F. Chan, S. Esedoglu, and F.E. Park, A fourth order dual method for staircase reduction 
in texture extraction and image restoration problems, Proc. 2010 IEEE 17th International Conference on Image Processing, Hongkong, 4137-4140, 2010.

[9] T. Chan, S. Esedoglu, F. Park, and A. Yip, Recent Developments in Total Variation Image Restoration, in Mathematical Models of Computer Vision, N. Paragios, Y. Chen, and O. Faugeras (Eds.), New York: Springer-Verlag, 2005.

[10] R.H. Chan and K. Chen, Multilevel algorithms for a Poisson noise removal model with total variation regularization, Int. J. Comput. Math., 84, 1183-1198, 2007.

[11] J. Frank, Three-Dimensional Electron Microscopy of Macromolecular Assemblies, Academic Press, 1996.

[12] M.A.T. Figueiredo and J.M. Bioucas-Dias, Deconvolution of Poissonian images using variable splitting and augmented Lagrangian optimization, "IEEE Workshop on Statistical Signal Processing", Cardiff, 2009.

[13] R. Glowinski and P. Le Tallec, Augmented Lagrangians and Operator-Splitting Methods in Nonlinear Mechanics, SIAM, Philadelphia, 1989.

[14] T. Goldstein and S. Osher, The split Bregman Method for L1 regularized problems, SIAM J. Imaging Sciences, 2, 323-343, 2009.

[15] S. Helgason, The Radon Transform, Birkhauser Boston, 1999.

[16] G.T. Herman, Image reconstruction from projections, Real-Time Imaging, 1, 3-18, 1995.

[17] W. Hinterberger and O. Scherzer, Variational methods on the space of functions of bounded Hessian for convexification and denoising, Computing, 76, 109-133, 2006.

[18] A.C. Kak and M. Slaney, Principles of Computerized Tomographic Imaging, IEEE Press, 1988.

[19] F. Knoll, K. Bredies, T. Pock, and R. Stollberger, Second order total generalized variation (TGV) for MRI, Magnetic Resonance in Medicine, 65(2), 480-491, 2011.

[20] T. Le, R. Chartrand, and T.J. Asaki, A variational approach to reconstructing images corrupted by Poisson noise, J. Math. Imaging Vision, 27, 257-263, 2007.

[21] M. Lysaker, A. Lundervold, and X.-C. Tai, Noise removal using fourth-order partial differential equation with applications to medical Magnetic Resonance Images in space and time, IEEE Trans. Image Process, 12, 1579-1590, 2003.

[22] D. Mumford and J. Shah, Optimal approximations by piecewise smooth functions and associated variational problems, Commun. Pure Appl. Math., 42, 577-685, 1989.

[23] F. Natterer, The Mathematics of Computerized Tomography, in Classics in Applied Mathematics, SIAM, 2001.

[24] V.Y. Panin, G.L. Zeng, and G.T. Gullberg, Total variation regulated EM algorithm [SPECT reconstruction], IEEE Trans. Nucl. Sci., 46, 2202-2210, 1999.

[25] R.S. Pantelic, R. Rothnagel, C.Y. Huang, D. Muller, D. Woolford, M.J. Landsberg, A. McDowall, B. Pailthorpe, P.R. Young, J. Banks, B. Hankamer, and G. Ericksson, The discriminative bilateral filter: An enhanced denoising filter for electron microscopy data, J. Structural Biology, 155, 395-408, 2006.

[26] C. Poschl and O. Scherzer, Characterization of minimizers of convex regularization functionals, in Frames and Operator Theory in Analysis and Signal Processing, Contemp. Math., AMS, Providence, RI, 451, 219-248, 2008.

[27] J. Radon, On the determination of functions from their integral values along certain manifolds, IEEE. Trans. Mecial Imaging, MI-5, 170-176, 1986.

[28] J. Rajan, K. Kannan, and M.R. Kaimal, An improved hybrid model for molecular image denoising, J Math. Imaging Vis., 31, 73-79, 2008.

[29] L. Rudin, S. Osher, and E. Fatemi, Nonlinear total variation based noise removal algorithms, Physica D, 60, 259-268, 1992.

[30] O. Scherzer, Denoising with higher order derivatives of bounded variation and an application to parameter estimation, Computing, 60, 1-27, 1998.

[31] S. Setzer and G. Steidl, Variational methods with higher order derivatives in image processing, M. Neamtu and L.L. Schumaker (Eds.), Approximation XII, Brentwood, Nashboro Press, 2008.

[32] S. Setzer, G. Steidl, and T. Teuber, Deblurring Poissonian images by split Bregman techniques, J. Visual Commun. Image Repres., 21, 193-199, 2010.

[33] S. Setzer, G. Steidl, and T. Teuber, Infimal convolution regularizations with discrete l1-type functionals, Commun. Math. Sci., 9(3), 797-827, 2011.

[34] E.Y. Sidky, C.M. Kao, and X. Pan, Accurate image reconstruction from few-views and limitedangle data in divergent-beam CT, J. X-Ray Sci. Tech., 14, 119-139, 2006.

[35] G. Steidl, A note on the dual treatment of higher-order regularization functionals, Computing, $76,135-148,2006$.

[36] X.C. Tai and C. Wu, Augmented Lagrangian method, dual methods and split Bregman iteration for ROF model, Scale Space and Variational Methods in Computer Vision, Second Interna- 
tional Conference, SSVM 2009, Voss, Norway, June 1-5, 2009. Proceedings. Lecture Notes in Computer Science 5567, 502-513, Springer, 2009.

[37] M. Unser and A. Aldroubi, A review of wavelets in biomedical applications, Proc. IEEE, 84(4), 626-638, 1996.

[38] D.Y. Wei and C.C. Yin, An optimized locally adaptive non-local means denoising filter for cryo-electron microscopy data, J. Structural Biology, 172(3), 211-218, 2010.

[39] C. Wu and X.C. Tai, Augmented Lagrangian method, dual methods, and split Bregman iteration for ROF, vectorial TV, and high order models, SIAM J. Imaging Sci., 3, 300-339, 2010.

[40] C. Wu, J. Zhang, and X.C. Tai, Augmented Lagrangian method for total variation restoration with non-quadratic fidelity, Inv. Prob. Imaging, 5, 237-261, 2011.

[41] Y.L. You and M. Kaveh, Fourth-order partial differential equations for noise removal, IEEE Trans. Image Process, 9, 1723-1730, 2000.

[42] R. Zanella, P. Boccacci, L. Zanni, and M. Bertero, Efficient gradient projection methods for edge-preserving removal of Poisson noise, Inv. Prob., 25, 045010, 2009. 